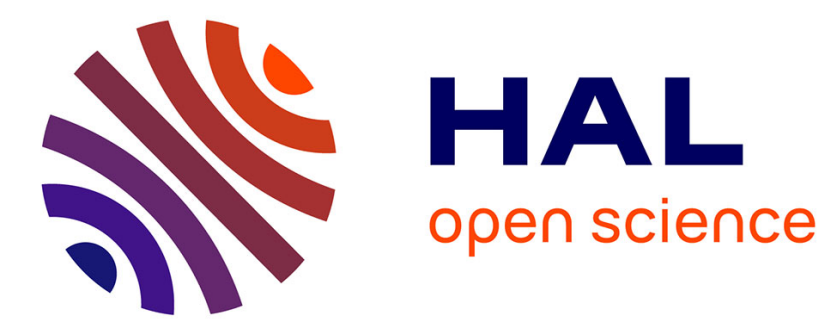

\title{
Spreading of superfluid drops
}

\author{
J.-F. Joanny
}

\section{To cite this version:}

J.-F. Joanny. Spreading of superfluid drops. Journal de Physique, 1985, 46 (5), pp.807-813. 10.1051/jphys:01985004605080700 . jpa-00210023

\section{HAL Id: jpa-00210023 https://hal.science/jpa-00210023}

Submitted on 1 Jan 1985

HAL is a multi-disciplinary open access archive for the deposit and dissemination of scientific research documents, whether they are published or not. The documents may come from teaching and research institutions in France or abroad, or from public or private research centers.
L'archive ouverte pluridisciplinaire HAL, est destinée au dépôt et à la diffusion de documents scientifiques de niveau recherche, publiés ou non, émanant des établissements d'enseignement et de recherche français ou étrangers, des laboratoires publics ou privés. 
Classification

Physics Abstracts

$67.70-68.10 \mathrm{G}$

\title{
Spreading of superfluid drops
}

\author{
J.-F. Joanny \\ Physique de la Matière Condensée, Collège de France, 11, Place Marcelin-Berthelot, 75231 Paris Cedex 05, France
}

(Reçu le 17 septembre 1984, révisé le 12 décembre, accepté le 8 janvier 1985)

\begin{abstract}
Résumé. — Nous étudions l'étalement d'une goutte d'hélium superfluide qui mouille totalement une surface solide plane à très basse température $(T \approx 0)$.

- Pour des gouttes macroscopiques, les forces dominantes sont les forces capillaires. a) Quand la règle d'Antonov est vérifiée (juste à la transition de mouillage), l'angle de contact dynamique est égal à l'angle de contact statique $\theta_{\mathrm{e}}=0$. A des temps d'étalement longs, la vitesse d'étalement atteint une valeur limite finie. Nous construisons un profil de goutte.self-similaire exact décrivant l'étalement. b) Dans des conditions de mouillage sec avec un pouvoir d'étalement $S>0$, nous ne savons pas exactement ce qui se passe mais nous donnons trois manières possibles de dissiper l'énergie capillaire : énergie cinétique, ondes capillaires, et vorticité.

- Pour des gouttes microscopiques, les forces dominantes sont les forces de van der Waals à longue portée. Nous proposons aussi un profil self-similaire de la goutte.
\end{abstract}

\begin{abstract}
We study the spreading of a superfluid helium drop totally wetting a smooth solid plane at very low temperatures $(T \approx 0)$.

- For macroscopic drops, the dominant forces are capillary forces. a) When Antonov's rule holds (i.e. when we are just at a wetting transition) the dynamic contact angle $\theta_{\mathrm{d}}$ is equal to the static contact angle $\theta_{\mathrm{e}}=0$, the spreading velocity reaches a finite value at long times. We construct an exact self-similar solution describing the spreading. b) In conditions of dry spreading with a spreading coefficient $S>0$ we do not know what will happen, but we list three different possibilities, depending on the ultimate fate of the capillary energy (kinetic energy, capillary waves, vorticity). finite.

- For microscopic drops, the dominant forces are the long range van der Waals forces. The spreading time is

For these two situations, we determine the self-similar profiles of the drop.
\end{abstract}

\section{Introduction.}

The dynamics of wetting phenomena has been recently extensively studied both experimentally and theoretically and is reasonably well understood at least when the surrounding medium is a vapour $[1,2]$. A droplet of a viscous liquid spreading on a plane surface can wet the solid either partially or totally.

In the case of partial wetting (when the static equilibrium contact angle $\theta_{\mathrm{e}}$ is finite) the drop reaches its equilibrium shape very rapidly $\left(\sim 10^{-5} \mathrm{~s}\right.$ for $1 \mathrm{~mm}$ radius drops) and in practice the spreading is not observable experimentally. More interesting is the case of total wetting when the static equilibrium contact angle is zero. For relatively small drops (smaller than the capillary length $\kappa^{-1}=\left(\frac{\rho g}{\gamma}\right)^{-1 / 2}$ ) we distinguish two regimes : a) A macroscopic regime in the centre of the drop where spreading is driven by capillary forces. In this regime the drop remains spherical and shows a finite dynamic contact angle $\theta_{\mathrm{d}}$ decreasing with time, related to the edge velocity $U$ by Tanner's law

$$
\theta_{\mathrm{d}} \sim\left[\frac{\eta U}{\gamma}\right]^{1 / 3}
$$

( $\gamma$ is the air liquid interfacial tension and $\eta$ the liquid viscosity). The macroscopic radius of the drop increases very slowly with time

$$
R(t) \sim t^{1 / 10}
$$

b) A microscopic regime (for simple organic liquids) near the drop edge where the spreading is not driven by capillary forces but by the long range van der Waals forces which become dominant for drop heights 
smaller than $1000 \AA$. This leads to the formation of a precursor film which advances ahead of the macroscopic droplet. The cross-over between the spherical shape and the macroscopic regime occurs at a distance $h \sim \theta_{\mathrm{d}}^{-2} a$ from the macroscopic edge, $a$ being some molecular distance.

At longer times, or for microscopic drops (smaller than $1 \mu \mathrm{m}$ ) van der Waals forces become dominant all over the drop and the spreading is much faster [3].

On the experimental side the study is limited by two major problems :

- the surface heterogeneities of the solid,

- the very long times involved in the spreading of these viscous drops. The complete spreading of a $1 \mathrm{~mm}$ drop would be, according to equation (2), as long as the age of the universe. The late stages of the spreading are not dominated by capillary forces but by van der Waals forces, however the characteristic times can be as long as half an hour.

Some data of Marmur and Tanner [2] in the macroscopic regime agree quite well with the theoretical predictions (Eqs. (1), (2)).

At very low temperatures $(T \ll 2 \mathrm{~K})$ liquid ${ }^{4} \mathrm{He}$ has two properties which makes its spreading properties very specific [4] :

- It becomes superfluid and, if the temperature is low enough, it can be considered as a perfect nonviscous fluid. The spreading times should then be considerably reduced compared to those of usual viscous fluids.

- The liquid-vapour surface tension $\gamma$ is very low $(\gamma \simeq 0.35 \mathrm{mN} / \mathrm{m})$ and liquid ${ }^{4} \mathrm{He}$ totally wets all the solids.

In this paper, we study the spreading of a small superfluid drop (smaller than the capillary length $\left.\kappa^{-1}=\left(\frac{\rho g}{\gamma}\right)^{-1 / 2}=0.5 \mathrm{~mm}\right)$ which totally wets the solid surface onto which it spreads. The study is limited to very low temperatures for two essential reasons :

- We neglect all dissipation. Dissipation exists at non-zero temperature even in the superfluid phase. It is usually agreed [5] that, in thin films, energy can be dissipated through the creation of vortices or of ripplons. This energy dissipation however becomes exponentially small at low temperatures.

- We study a hydrodynamic spreading, neglecting evaporation and recondensation processes. This is legitimate when the vapour pressure is very low (at zero temperature).

In practice, in the presence of helium vapour there always exists a thin liquid film spread on the solid plane [6]. A drop thus spreads on this thin film. At very low vapour pressure (for under-saturated films) the thickness of this film can be reduced to a few molecular thicknesses and this film has no effect on the drop-spreading. We restrict our study to this limit.
In the following, we will ignore the existence of this film. In the language of reference [7] we study here superfluid spreading in the dry case. The study is limited here to situations where the Antonov rule holds, when the spreading power $S$ is either negative or zero.

We first discuss the lubrication approximation for a superfluid drop. We then study the spreading of a drop driven by capillary forces, by gravity forces, and by the long-range component of van der Waals forces. The last section is a discussion of the relative importance of these forces.

\section{The lubrication approximation for a perfect non- viscous fluid.}

2.1 ONE-DIMENSIONAL DROP. - For pedagogical purposes we first study the spreading of a one-dimensional drop (or an infinitely long drop spreading in one dimension). The shape of the drop is given by the height $\zeta(x, t)$ at a distance $x$ from the centre. At each point the velocity $\mathbf{v}$ is a two-dimensional vector in the plane of the drop.

In the lubrication approximation, we neglect the variation with height of the $x$ component of the velocity $v$ parallel to the plane.

The vertical component of the velocity $w$ is then, according to the continuity equation,

$$
w=-z \frac{\mathrm{d} v}{\mathrm{~d} x} .
$$

In the late stages of the spreading the maximum height $\zeta$ is very small and the downward velocity is negligible.

The pressure at a height $z$ is related to the external potential $W(z)$ (either gravity or van der Waals) by

$$
P(z)=P(\zeta)+W(\zeta)-W(z) .
$$

The pressure just below the drop interface $P(\zeta)$ is related to the external pressure by the Laplace equation

$$
P(\zeta)=P_{\mathrm{ext}}+C \gamma \approx P_{\mathrm{ext}}-\gamma \Delta \zeta .
$$

The velocity $v$ being independent of height, it is convenient to cut the drop into small vertical slices of volume $\zeta \mathrm{d} x$ moving with velocity $v$.

The forces acting on such a « slice " inside the drop are (Fig. 1) :

- Two pressure forces on the vertical edges of the slice

$$
F_{\mathrm{p}}=\left\{\left[\frac{-\mathrm{d}}{\mathrm{d} x}(\zeta(P(\zeta)+W(\zeta)))\right]+W(\zeta) \frac{\partial \zeta}{\partial x}\right\} \mathrm{d} x .
$$

- Two pressure forces on the interfaces vapourliquid and solid-liquid

$$
F_{\mathrm{p}}^{\prime}=\left[P_{\text {ext }} \frac{\partial}{\partial x} \zeta\right] \mathrm{d} x
$$




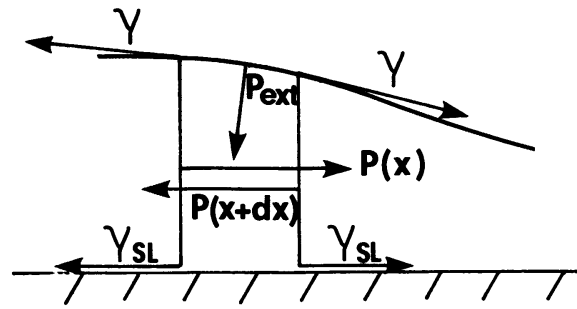

Fig. 1. - Forces acting on a small vertical slice inside the superfluid drop. liquid

- Two capillary forces at the interface vapour$F_{\mathrm{c}}=\left[+C \gamma \frac{\partial \zeta}{\partial x}\right] \mathrm{d} x C$ being the total curvature of the interface $C \approx-\Delta \zeta$.

- Two capillary forces at the interfaces solid-liquid which cancel. then

The equation of motion of such a slice of liquid gives

$$
\rho \zeta\left[\frac{\partial}{\partial t} v+v \frac{\partial v}{\partial x}\right]=-\zeta \frac{\partial W(\zeta)}{\partial x}-\zeta \frac{\partial P(\zeta)}{\partial x} .
$$

This equation of motion has to be modified for the last « slice » containing the edge (Fig. 2) :

- There is just one capillary force at the liquidvapour interface : $-\gamma \cos \theta$.

- The two forces at the solid-liquid interface do not cancel. They give a contribution $\gamma_{S v}-\gamma_{L S}$.

The total force balance on the edge is :

$$
-\gamma \cos \theta_{\mathrm{d}}+\gamma_{\mathrm{sv}}-\gamma_{\mathrm{LS}}=0 \text {. }
$$

(The acceleration term is negligible when the width of the slice $\mathrm{d} x$ goes to zero.)

Equation (7) is the central result of this paper : the dynamic contact angle $\theta_{\mathrm{d}}$ is related to the three interfacial tensions by the usual Young equation. This means that for a non-viscous fluid, the dynamic contact angle is equal to the static contact angle. This result is true in any geometry. It is very different from the dynamic contact angle of viscous liquids which strongly depends on the velocity of the advancing interface (cf. Eq. (1)). For viscous liquids one should also take the viscous force into account in equa-

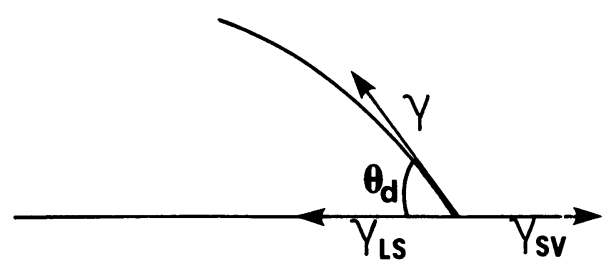

Fig. 2. - Forces acting on the edge of the superfluid drop. tion (7). In the edge the velocity gradients become very large, the viscous force has a singularity at the edge and contributes to the force balance. This «dynamic Young equation " can be satisfied only if $\gamma_{\mathbf{s v}}-\gamma_{\mathbf{L S}}-$ $\gamma \leqslant 0$, otherwise $\cos \theta_{\mathrm{d}}$ would be larger than one. In terms of the spreading power $S=\gamma_{S V}-\gamma_{L S}-\gamma$, the dynamic Young equation is valid within two limits :

i) $S<0$ for partially wetting superfluids;

ii) $S=0$ in the case where the fluid is totally wetting the solid.

The other case of a totally wetting fluid with a positive spreading power $S$ will not be considered here. We will discuss it briefly in the conclusion.

We can rewrite equation (6a) introducing the singularities at the edges $x= \pm R$.

$$
\begin{aligned}
& \rho \zeta\left[\frac{\partial}{\partial t} v+v \frac{\partial v}{\partial x}\right]=-\zeta \frac{\partial W(\zeta)}{\partial x}-\zeta \frac{\partial P(\zeta)}{\partial x}+ \\
& \quad+[\delta(x-R)-\delta(x+R)]\left(\gamma_{\mathrm{sv}}-\gamma_{\mathrm{SL}}-\gamma \cos \theta_{\mathrm{d}}\right)
\end{aligned}
$$

Energy conservation can be checked by multiplying equation (6b) by $v$ and integrating from $-R$ to $+R$ we get

$$
\begin{aligned}
\frac{\mathrm{d}}{\mathrm{d} t} \int \rho \frac{\zeta v^{2}}{2} \mathrm{~d} x= & \frac{3}{2} \gamma \int\left(\frac{\mathrm{d} \zeta}{\mathrm{d} x}\right)^{2} \frac{\partial v}{\partial x} \mathrm{~d} x-\left(\gamma+\gamma_{\mathrm{LS}}-\gamma_{\mathrm{SV}}\right) \times \\
& \times \int \frac{\mathrm{d} v}{\mathrm{~d} x} \mathrm{~d} x-\frac{\mathrm{d}}{\mathrm{d} t} \int \mathrm{d} x \int_{0}^{\zeta} \mathrm{d} z W(z)
\end{aligned}
$$

The left-hand side is the time derivative of the kinetic energy. One can directly check that the right-hand side is the opposite of the time derivative of the total interfacial energy (on both interfaces). The last term is the variation of potential energy.

2.2 TWO-DIMENSIONAL DROP. - For the more realistic two-dimensional drop, the same analysis can be carried out on a small angular sector of width $\mathrm{d} \theta$ between $^{\circ} r$ and $r+\mathrm{d} r$.

The full equation of motion for the velocity is then

$$
\begin{aligned}
\rho \zeta\left[\frac{\partial v}{\partial t}+v \frac{\partial v}{\partial r}\right] & =-\zeta \frac{\partial W(\zeta)}{\partial r}-\zeta \frac{\partial P(\zeta)}{\partial r}+ \\
& +\delta(r-R)\left(\gamma_{\mathrm{Sv}}-\gamma_{\mathrm{SL}}-\gamma \cos \theta_{\mathrm{d}}\right)
\end{aligned}
$$

The energy conservation is obtained again by multiplying this momentum equation by the velocity and integrating over the drop surface.

\section{Spreading driven by capillary forces.}

In this section, we neglect the effect of any external potential, and study the spreading of a superfluid helium drop due only to capillary forces. We set $W(\zeta)=0$ in equation (9). 


\subsection{ENERGY CONSERVATION.}

3.1.1 Partially wetting drop : $\theta_{\mathrm{e}} \neq 0, S<0$. - At the beginning of the spreading, the drop is spherical with a radius $\boldsymbol{R}_{\mathbf{0}}$. Its energy is only capillary. As the drop spreads, the capillary energy decreases and the kinetic energy increases. When the capillary energy is minimum, the kinetic energy is non-zero and the radius continues to increase : the drop oscillates back and forth around the equilibrium shape : a spherical cap with a contact angle $\left(\theta_{\mathrm{e}} \neq 0\right)$ and a radius $R_{\mathrm{eq}}$.

Calling $\zeta=\zeta_{0}(R)$ this spherical cap we can linearize the equation of motion around this equilibrium state $\zeta(r, t)=\zeta_{0}(r)+\delta \zeta(r, t)$

The equation of motion (9) gives

$$
\rho \frac{\partial v}{\partial t}=\gamma \frac{\mathrm{d}}{\mathrm{d} r} \Delta \delta \zeta,\left.\quad \frac{\partial}{\partial r} \delta \zeta\right|_{r=R}=0 .
$$

The mass conservation equation in the lubrication approximation can be written

$$
\operatorname{div} \zeta v+\frac{\partial \zeta}{\partial t}=0
$$

or after linearization

$$
\operatorname{div} \zeta_{0} v+\frac{\partial \delta \zeta}{\partial t}=0
$$

Looking for a periodic solution for $v$ and $\delta \zeta$ of pulsation $\omega$, we get

$$
-\omega^{2} \delta \zeta+\operatorname{div}\left[\frac{\gamma}{\rho} \zeta_{0} \operatorname{grad} \Delta \delta \zeta\right]=0 .
$$

The perturbation $\delta \zeta$ varies over one radius $R_{\mathrm{eq}}$ of the drop at equilibrium and thus

$$
\omega^{2} \sim \frac{\theta_{\mathrm{e}}}{\rho R_{\mathrm{eq}}^{3}} \gamma .
$$

In pratice, partial wetting is not found : as far as we know superfluid helium wets all solids.

3.1.2 Totally wetting drop : $S=0$. - In the more interesting case of a solid totally wetted, the drop spreads and makes a thin helium film between the solid and the vapour phase. For this film the capillary energy vanishes and, at very long times, the kinetic energy reaches a finite value equal to the initial capillary energy. Consequently, the velocity of the interface reaches a finite value and the radius of the drop increases linearly with time

$$
R(t)=v_{\infty} i
$$

The velocity $v_{\infty}$ is fixed by energy conservation

$$
v_{\infty} \sim\left(\frac{\gamma}{\rho R_{0}}\right)^{1 / 2} .
$$

3.2 DROP SHAPE. - We now study the shape of a totally wetting superfluid helium drop in greater detail. The two basic equations are the force balance (9) and the mass conservation equation (10). We look for self-similar solutions for the velocity $v(r, t)$ and the interface $\zeta(r, t)$ :

$$
\begin{aligned}
& v(r, t)=v_{0}(t) g\left[\frac{r}{R(t)}\right] \\
& \zeta(r, t)=h(t) f\left[\frac{r}{R(t)}\right]
\end{aligned}
$$

$R(t)$ is the drop radius, $h(t)$ is the height of the drop in the centre and $v_{0}(t)$ is the velocity of the edge related to the radius $R$ by $v_{0}(t)=\frac{\mathrm{d} R}{\mathrm{~d} t}$.

With these definitions we have the following boundary conditions on $f$ and $g$

$$
\left.\begin{array}{ll}
f(1)=0 & f(0)=1 \\
g(1)=1 & g(0)=0 .
\end{array}\right\}
$$

The contact angle is the equilibrium contact angle $\theta_{\mathrm{e}}=0$ :

$$
f^{\prime}(1)=0 \text {. }
$$

The global volume of the drop is conserved in the spreading

$$
2 \pi R^{2} h \int_{0}^{1} u f(u) \mathrm{d} u=\Omega
$$

The product $R^{2} h=\Omega^{\prime}$ is thus time independent. Inserting the self-similar solutions in the equation of motion, we get an equation for the radius $R$

$$
R^{\prime \prime}(t)=\frac{\alpha}{R^{5}}
$$

where $\alpha$ is an integration constant.

The general solution of this equation at long times is

$$
\begin{aligned}
v & =v_{\infty}\left[1-\frac{\alpha}{4\left(v_{\infty} t\right)^{4} v_{\infty}^{2}}\right] \\
R & =v_{\infty} t\left[1+\frac{\alpha}{12\left(v_{\infty} t\right)^{4} v_{\infty}^{2}}\right]+\text { const. } \\
h(t) & =\frac{\Omega^{\prime}}{R^{2}} \cong \frac{\Omega^{\prime}}{\left(v_{\infty} t\right)^{2}} .
\end{aligned}
$$

At long times the velocity reaches a finite value $v_{\infty}$ and the radius increases linearly with time. The velocity increases with time, the integration constant $\alpha$ is positive.

The dimensionless functions $f(u)$ and $g(u)$ satisfy 
a system of coupled differential equations

$$
\left.\begin{array}{l}
\frac{1}{u} \frac{\mathrm{d}}{\mathrm{d} u}(f g u)=\frac{1}{u} \frac{\mathrm{d}}{\mathrm{d} u}\left(f u^{2}\right) \\
\frac{\rho \alpha}{\Omega^{\prime} \gamma} g(u)=\frac{\mathrm{d}}{\mathrm{d} u}\left[\frac{\mathrm{d}^{2}}{\mathrm{~d} u^{2}}+\frac{1}{u} \frac{\mathrm{d}}{\mathrm{d} u}\right] f .
\end{array}\right\}
$$

The solution of these equations satisfying the boundary conditions $(15 \mathrm{a}, 15 \mathrm{~b})$ is

$$
\left.\begin{array}{l}
g(u)=u \\
f(u)=u^{4}-2 u^{2}+1
\end{array}\right\} u=\frac{r}{R}
$$

with $\alpha=\frac{32 \gamma \Omega^{\prime}}{\rho}$.

The volume conservation (16) determines the cons$\operatorname{tant} \Omega^{\prime}=\frac{3}{\pi} \Omega$. The velocity varies linearly with radius and the shape of the drop is not spherical. It is sketched in figure 3.

The last constant to be determined is the spreading velocity $v_{\infty}$. Energy conservation gives

$$
\frac{1}{2} \rho v_{\infty}^{2} 2 \pi \Omega^{\prime} \int_{0}^{1} u^{3} f(u) \mathrm{d} u=4 \pi \gamma R_{0}^{2}
$$

( $R_{0}$ is the initial drop radius $\Omega=\frac{4}{3} \pi R_{0}^{3}$ ).

The spreading velocity is then

$$
v_{\infty}=\left(\frac{24 \gamma}{\rho R_{0}}\right)^{1 / 2}
$$

\section{Gravity effects.}

Even for the spreading of a drop smaller than the capillary length $\kappa^{-1}$, gravity effects become important at longer times. This can be checked by comparing the gravity and capillary terms in the equation of motion (9). The order of magnitude of the capillary term $\zeta \frac{\partial P(\zeta)}{\partial r}$ is $\gamma \frac{h^{2}}{R^{3}}$. The order of magnitude of the

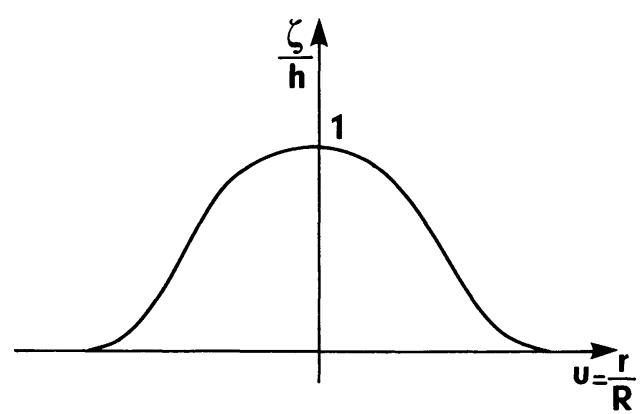

Fig. 3. - Shape of a superfluid drop spreading under the action of capillary forces. gravity term $\zeta \frac{\partial W}{\partial r}(W=\rho g \zeta)$ is $\rho \frac{h^{2}}{R}$. The gravity term becomes dominant as soon as $\kappa^{2} R^{2} \gg 1$ : when the radius of the drop becomes larger than the capillary length $\kappa^{-1}$. This always occurs for a totally wetting drop $\left(\theta_{\mathrm{e}}=0\right)$. In order to study the spreading driven by gravity of a totally wetting superfluid drop we use the continuity equation (10) and the equation of motion (9), neglecting the capillary term, with a potential $W(\zeta)=\rho g \zeta$.

Looking for a self-similar solution in the form (14), we find

$$
\begin{aligned}
& v_{0}(t)=v_{\infty}\left[1-\frac{2 g \Omega}{\pi\left(v_{\infty} t\right)^{2} v_{\infty}^{2}}\right] \\
& R(t)=v_{\infty} t\left[1+\frac{2 g \Omega}{\pi\left(v_{\infty} t\right)^{2} v_{\infty}^{2}}\right]+\text { const. } \\
& h(t)=\frac{2}{\pi} \frac{\Omega}{\left(v_{\infty} t\right)^{2}} .
\end{aligned}
$$

At very long times the velocity reaches a finite limit : all the gravitational energy has become kinetic energy.

The radius increases linearly with time.

The velocity profile is still linear $g(u)=u\left(u=\frac{r}{R}\right)$.

The drop shape is given by $f(u)=1-u^{2}(23)$ and is sketched in figure 4.

If we totally neglect capillary forces, there is no way to satisfy Young equation (7) for the dynamic contact angle $\theta_{\mathrm{d}}=0$. Capillary forces are thus dominant in a small region of size $l$ near the drop edge. The size of this region can be estimated by comparing more precisely the capillary and gravity terms in the equations of motion.

At a distance $l$ from the edge, the gravity term is

$$
\left.\rho g \frac{\partial \zeta}{\partial r}\right|_{R-l}=\frac{\rho g h(t)}{R(t)} f^{\prime}(1) .
$$

The capillary term is $\gamma \frac{\mathrm{d}}{\mathrm{d} r} \Delta \zeta(r) \sim \gamma \frac{\zeta(l)}{l^{3}} \approx \gamma \frac{h}{l^{3}} \times$ $f\left(1-\frac{l \cdot}{R}\right)=\gamma \frac{h}{R l^{2}}$ and thus $\kappa^{2} l^{2} \approx 1$. Capillary forces dominate gravity forces over one capillary length around the drop edge (Fig. 4).

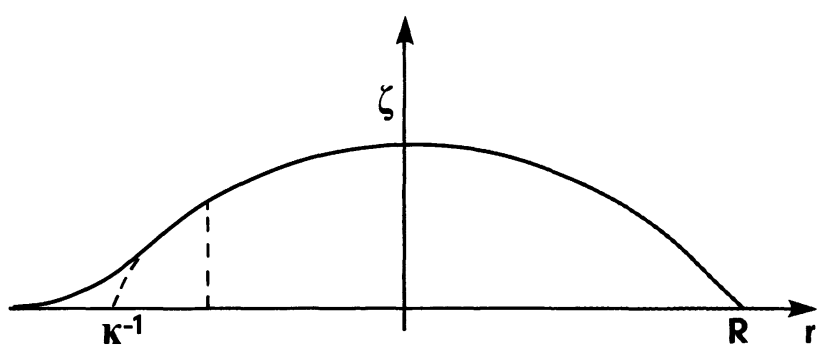

Fig. 4. - Shape of a superfluid drop spreading under the action of gravity forces. Capillary forces are dominant in a small region of size $\kappa^{-1}$ around the edge. 


\section{Spreading driven by van der Waals forces.}

At very long times, the thickness of a totally wetting drop becomes very small. When it becomes smaller than a few thousand Angströms, the dominant forces are neither the gravity forces nor the capillary forces but the long-range component of van der Waals forces. These forces are strongly attractive and the spreading velocity is considerably increased.

For microscopic drops (of size smaller than $1 \mu \mathrm{m}$ ) the spreading is dominated by van der Waals forces at all times.

At long times the height $\zeta(x, t)$ of the drop becomes very small and the van der Waals potential

$$
W(\zeta)=-\frac{A}{6 \pi \zeta^{3}}
$$

( $A$ being a Hamaker constant) becomes very large. The total energy being constant, in this regime. The kinetic energy can become very large and the spreading velocity $v_{0}(t)$ increases with time, it does not reach a finite limit.

To describe this regime, we again use the continuity equation (10) and the equation of motion, with the van der Waals potential.

Looking for a self-similar solution in the form (14) we find

$$
\left.\begin{array}{rl}
v_{0}(t) & =\frac{R_{0}}{2 t_{\infty}} \frac{1}{\left(1-t / t_{\infty}\right)^{3 / 2}} \\
R(t) & =R_{0} \frac{1}{\left(1-t / t_{\infty}\right)^{1 / 2}} \\
h(t) & =h_{0}\left(1-t / t_{\infty}\right)
\end{array}\right\}
$$

$R_{0}$ and $h_{0}$ are the initial radius and height of the drop.

The important result in this regime is that the spreading time is finite. After a time $t_{\infty}, h=0$ and an infinitely thin helium film has formed between the solid and the vapour.

The dimensionless velocity is still a linear function of the position $: g(u)=u$.

The shape of the drop is defined by

$$
f(u)=\left[1+u^{2}\left(\frac{9 \pi h_{0}^{3} R_{0}^{2} \rho}{4 A t_{\infty}^{2}}\right)\right]^{-1 / 3}\left(u=\frac{r}{R}\right) .
$$

The drop profile has here an infinitely long tail and $R_{0}$ is no longer the radius of the drop but the width of the profile. In reduced units this width is 1 and

$$
t_{\infty}^{2}=\frac{9 \pi}{4} \frac{\rho R_{0}^{2} h_{0}^{3}}{A} .
$$

With reasonable orders of magnitude [4-6] $\rho=$ $0.14 \mathrm{cgs}, A=10^{-15} \mathrm{erg}$ (a value deduced from thin films thickness) $R_{0}=h_{0}=1 \mu \mathrm{m}, t_{\infty} \approx 10^{-3} \mathrm{~s}$.

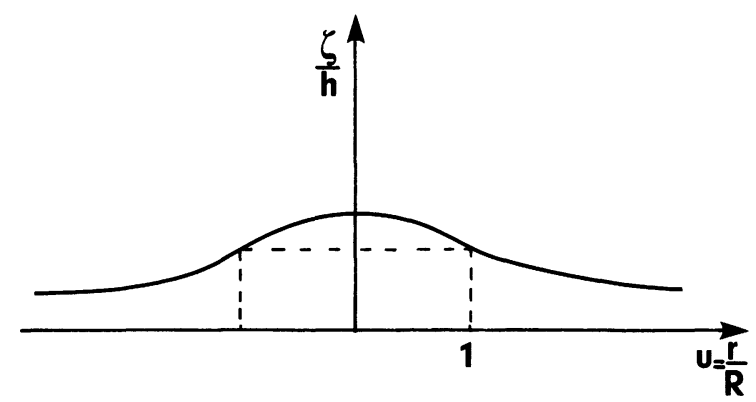

Fig. 5. - Shape of a superfluid drop spreading under the action of van der Waals forces.

Of course there are physical cut-offs to the shape of the drop given by equation (26), for instance the minimum film thickness is of the order of magnitude of the atomic length scale.

\section{Discussion.}

6.1 The PRECURSOR FILM. - For superfluid liquids as well as for viscous liquids, the spreading of a drop can be separated into two important regimes :

- A macroscopic regime where spreading is driven either by capillary or by gravity forces. The two important results in this regime are the equality between static and dynamic contact angles and the constant spreading velocity;

- A microscopic regime dominated by van der Waals forces. The important result being that the complete spreading of the drop is done in a finite time $t_{\infty}$.

In the analysis of the macroscopic regime, we have neglected the long-range component of van der Waals forces. This is only legitimate in regions where the drop is thick enough. In particular it cannot be legitimate near the drop edge where the helium thickness goes to zero. In this region van der Waals forces dominate. Their effect has been discussed by de Gennes in the case of viscous fluids [1]. He has shown that these long-range attractive forces tend to thicken the liquid film and are responsible for the creation of a precursor film advancing ahead of the macroscopic drop. Such a precursor film should also exist for superfluids. We do not want to discuss here the precise sha"pe of such a film but we can simply discuss the cross-over between the film and the macroscopic drop, for instance when spreading is dominated by capillarity. We first neglect van der Waals forces and get the macroscopic shape [20]. We then compare the van der Waals and capillary terms in the equation of motion. The cross-over occurs at a distance $b$ from the drop edge, at this point $\zeta(r)$ and $\frac{\mathrm{d} \zeta}{\mathrm{d} r}$ are continuous.

The capillary term is $\left.\gamma \frac{\mathrm{d}}{\mathrm{d} r} \Delta \zeta\right|_{r=R-b} \approx \gamma \frac{h}{R^{3}}$. 
The van der Waals term is

$$
\begin{aligned}
&\left.\frac{A}{2 \pi \zeta^{4}(R-l)} \frac{\partial \zeta}{\partial r}\right|_{R-b}=\frac{A}{6 \pi} \frac{h}{R h^{4}} f^{\prime}\left(1-\frac{b}{R}\right) \times \\
& \times \frac{1}{f^{4}\left(1-\frac{b}{R}\right)}
\end{aligned}
$$

According to equation (20) $f\left(1-\frac{b}{R}\right) \sim \frac{b^{2}}{R^{2}}$ and $f^{\prime}\left(1-\frac{b}{R}\right) \sim \frac{b}{R}$.

The van der Waals term is then $: \frac{A R^{6}}{6 \pi h^{3} b^{7}}$.

At the cross-over these two terms are equal and

$$
b=\left[\frac{A R^{9}}{6 \pi \gamma h^{4}}\right]^{1 / 7} \sim \frac{a^{2 / 7}}{\Omega^{4 / 7}}\left(v_{\infty} t\right)^{17 / 7} .
$$

We have here introduced the drop volume $\Omega=h R$ and an atomic length $a^{2}=\frac{A}{6 \pi \gamma}$.

At short times this length $b$ is smaller than the drop radius but it increases faster. Van der Waals forces dominate the spreading when $b$ becomes equal to $R$.

6.2 Conclusion. - On the experimental part, the central result of this paper seems to be the equality between static and dynamic macroscopic contact angles for a superfluid. It should be independent on the geometry and could be tested either on spreading helium drops or on advancing films.

This result could also be important for the explanation of the hysteresis effect appearing in helium flows in porous media. Part of this hysteresis might be related to contact angle hysteresis. For superfluids, contact angle hysteresis has only a static origin (for viscous fluids there can be a static and a dynamic effect) : the contact angle is modified locally by the heterogeneities of the solid surface.

Finally, we must stress the limitations of this study :

- it is restricted to zero temperature when dissipation and recondensation phenomena are negligible;

- all the drop profiles calculated here are selfsimilar profiles. It is not obvious that, whatever the initial conditions, a spreading superfluid drop has a self-similar shape. We have not checked the stability of the calculated shapes. In particular it is likely that, in the microscopic regime, the shape of a superfluidvapour interface depends strongly on the initial conditions ;

- for totally wetting fluids, the study has been limited to the $S=0$ limit. If the spreading power $S$ is positive, we were not able to find a self-similar solution to the Navier-Stokes equation (9), but, if we assume that all the capillary energy is transformed into kinetic energy, the energy conservation in the macroscopic regime leads to a time-variation of the drop radius :

$$
R(t)=R(0) \exp \left[\alpha\left(\frac{S}{\rho \Omega}\right)^{1 / 2} t\right]\left(^{1}\right)
$$

$\alpha$ is a numerical constant, $\Omega$ the drop volume and $\rho$ its density. However, many other physical phenomena can occur and part of the capillary energy can be transformed into vorticity or capillary waves, in which case the radius variation is slower than (29).

\section{Acknowledgments.}

I am very grateful to P. G. de Gennes for suggesting this problem and for numerous discussions and to D. Salin for introducing me to helium films.

$\left.{ }^{1}\right)$ This law was independently proposed by one anonymous referee.

\section{References}

[1] a. De Gennes, P. G., C. R. Hebd. Séan. Acad. Sci. 298 (1984) 111.

b. Lopez, J., Miller, C. A., Ruckenstein, E., Colloid Interface Sci. 56 (1976) 460.

[2] Marmur, A., Adv. Colloid Interface Sci. 19 (1982) 75. TANNER, L. H., J. Phys. D 12 (1979) 1473.

[3] De Gennes, P. G., C. R. Hebd. Séan. Acad. Sci. 298 (1984) 475.

[4] WILKs, J., The properties of solid and liquid helium (Oxford University Press) 1967.
[5] LANGer, J., RePPy, J., in Progress in low temperature physics, vol. VI, p. 1, C. J. Gorter Editor (North Holland, Amsterdam) 1972.

[6] BREWER, D. F., in the Physics of solid and liquid helium, Benneman K. H. and Ketterson J. B. editors (Wiley and Son, New York) 1978.

[7] Joanny, J. F., De Gennes, P. G., C. R. Hebd. Séan. Acad. Sci. 299 (1984) 279. 